\title{
Modificação de Polímeros Termorrígidos por Separação de Fases Induzida por Reação Química. Sistema Éter Diglicidílico do Bisfenol-A e Trietilenotetramina com Copolímeros Acrílicos
}

\author{
Filiberto González Garcia \\ Instituto de Ciências Exatas, Universidade Federal de Itajubá
}

\author{
Bluma G. Soares \\ Instituto de Macromoléculas Prof. Eloisa Mano, UFRJ
}

\author{
Alessandra F. Neves, Márcia G. de Oliveira \\ Laboratório de Tecnologia de Materiais Poliméricos, Instituto Nacional de Tecnologia
}

\begin{abstract}
Resumo: O comportamento da separação de fases e da gelificação do sistema do éter diglicidílico do Bisfenol-A com trietilenotetramina modificado com diferentes copolímeros acrílicos foi estudado. As massas moleculares e as concentrações de grupos carboxílicos nos copolímeros provocaram mudanças significativas na morfologia e provocaram ligeiras mudanças para a observação da separação de fases. Contudo, não mudaram de maneira significativa os tempos de gelificação e não afetaram a velocidade da reação. O sistema modificado com os copolímeros acrílicos mostrou o efeito de retardação cinética. A morfologia foi relacionada com a aderência ao cisalhamento, em juntas de aço-aço, através de ensaios de resistência mecânica usando juntas de cisalhamento simples, as que foram produzidas com o sistema modificado com os copolímeros acrílicos segundo a norma ASTM D 1002. Amostras com a fase dispersa apresentando morfologia com partículas de diâmetro médio menor que $0,10 \mu \mathrm{m}$ mostraram o melhor desempenho de aderência ao cisalhamento para as massas moleculares dos copolímeros estudadas. Entretanto, um melhor comportamento mecânico para as concentrações de grupos carboxílicos nos copolímeros acrílicos foi observado para a morfologia com distribuição de tamanhos de partículas na faixa de 0,20 a $0,52 \mu \mathrm{m}$.
\end{abstract}

Palavras-chave: Éter diglicidílico do Bisfenol-A, trietilenoamina, copolímeros acrílicos, separação de fases induzida por reação química.

\section{Modification of Thermosetting Polymers by Induced Phase Separation by Chemical Reaction. Diglycidyl Ether of Bisphenol-A and Triethylenetetramine with Acrylic Copolymers}

\begin{abstract}
The cloud point and the gel time behavior of an epoxy system based on diglycidyl ether of Bisphenol-A with triethylenetetramine modified with different acrylic copolymers were studied. The molecular weights and the concentration of carboxyl groups in the copolymers affected the morphology and the cloud point, but did not affect the gel times and reaction rates significantly. The system modified with the acrylic copolymers exhibited kinetic retardation effects. The morphology was related to adherence to the lap shear in steel-steel joints, through mechanical resistance essays using a single-lap-joint, which was produced with the system modified with the acrylic copolymers according to ASTM D 1002. The best performance in adherence to the single lap shear for the molecular weights studied was obtained with a morphology based on particles with average diameters lower than $0.10 \mu \mathrm{m}$. However, the best mechanical behavior for the concentration of carboxylic groups was achieved when the morphology was based on a particles size in the range from 0.20 to $0.52 \mu \mathrm{m}$.
\end{abstract}

Keywords: Diglycidyl ether of Bisfenol-A, triethylenetetramine, acrylic copolymers, induced phase separation by chemical reaction.

\section{Introdução}

As resinas epoxídicas constituem uma das mais importantes classes de polímeros termorrígidos utilizados em adesivos, matrizes para compósitos reforçados e recobrimentos. Estes materiais apresentam dureza, excelente adesão, resistência química e baixa contração, como resultado da natureza estrutural dos monômeros que formaram a rede tridimensional. No entanto, quando estes materiais são destinados a aplicações que solicitam alta resistência mecânica, é comum a incorporação de modificadores que originem uma segunda fase e propiciem uma tenacificação.

Autor para correspondência: Filiberto González Garcia, Departamento de Física e Química, Instituto de Ciências Exatas, Universidade Federal de Itajubá (UNIFEI), Campus Prof. José Rodrigues Seabra, Avenida BPS 1303 - Bairro Pinheirinho, CEP: 37500-903, Itajubá, MG, Brasil. E-mail: fili@unifei.edu.br 
A dispersão da segunda fase no polímero termorrígido pelo método de separação de fases induzida por reação química apresenta como vantagem a estabilidade das partículas dispersas e a possibilidade de se obter partículas com diferentes características morfológicas. Isto pode ser possível pelo controle dos fatores cinéticos e termodinâmicos envolvidos na reação química ${ }^{[1]}$.

Para a modificação de polímeros termorrígidos pelo método de separação de fases induzida por reação química é possível utilizar como modificadores, tanto elastômeros como termoplásticos, tendo como condição única, que sejam solúveis nos monômero iniciais e durante a reação química ocorra a precipitação do modificador, isto é, que o modificador separe de fases durante a polimerização ${ }^{[1]}$.

A utilização de termoplásticos como modificadores de polímeros termorrígidos é bem conhecida. Em particular, o emprego de poli(metacrilato de metila) (PMMA) tanto na forma de homopolímero ${ }^{[2-10]}$ como de copolímeros aleatórios ${ }^{[1]}$, graftizados $^{[12,13]}$ ou em bloco ${ }^{[14]}$ são de grande interesse, pois o PMMA é solúvel na resina epoxídica do tipo éter diglicídico do Bisfenol-A (DGEBA) e não reage quimicamente com ela $^{[2,6,15]}$. Recentemente foi relatada como alternativa para conseguir uma dispersão estável de partículas elastoméricas no polímero termorrígido o emprego de copolímeros aleatórios baseados em acrilato de 2-etilhexila (AEH) e metacrilato de metila (MMA), onde pela concentração de MMA, e o tipo de endurecedor é controlada a separação de fases e por tanto a morfologia e as propriedades mecânicas ${ }^{[11,16]}$. Recentemente também, foram divulgados dois trabalhos onde são utilizados copolímeros triblocos que geram morfologias nanoestruturadas, pelo emprego de segmentos com solubilidades diferentes para propósitos de tenacificação de polímeros termorrígidos ${ }^{[17,18]}$.

Neste trabalho estuda-se o sistema epoxídico constituído pelo éter diglicidílico do Bisfenol-A com trietilenotetramina modificado com diferentes copolímeros acrílicos, com o objetivo de obter informação do comportamento morfológico induzido por reação química por mudanças na massa molecular e a concentração de grupos carboxílicos no modificador. No trabalho se relaciona a morfologia gerada por reação química com a aderência ao cisalhamento de juntas do tipo aço-aço semelhante à liga metálica utilizada nos dutos de distribuição de petróleo e gás natural e encontra-se a influência dos copolímeros acrílicos na velocidade da reação do sistema epoxídico.

\section{Experimental}

\section{Materiais}

O monômero epoxídico utilizado foi a resina líquida do tipo éter diglicidílico do Bisfenol-A, (DGEBA) produto DER 331 da Dow Química do Brasil com 187,5 g eq ${ }^{-1}$ o que foi determinado por titulação química usando brometo de hidrogênio produzido "in situ" segundo a metodologia descrita na
Tabela 1. Propriedades dos copolímeros acrílicos.

\begin{tabular}{|c|c|c|c|}
\hline Copolímero & $\begin{array}{c}\overline{\mathrm{Mn}} \\
(\mathrm{SEC})\end{array}$ & $\begin{array}{c}\text { Concentração de } \\
\text { grupos carboxílicos } \\
\left(\mathrm{mmol} \mathrm{g}^{-1}\right)\end{array}$ & $\begin{array}{c}\text { Funcionalidade } \\
\left.\text { F (eq. } \text { mol }^{-1}\right)\end{array}$ \\
\hline $\mathrm{M}_{1}$ & $8,74 \times 10^{3}$ & $1,12 \times 10^{-1}$ & 0,98 \\
\hline $\mathrm{M}_{2}$ & $3,40 \times 10^{3}$ & $2,91 \times 10^{-1}$ & 0,99 \\
\hline $\mathrm{M}_{3}$ & $2,10 \times 10^{3}$ & $4,57 \times 10^{-1}$ & 0,96 \\
\hline $\mathrm{A}_{1}$ & $1,19 \times 10^{4}$ & 0,00 & - \\
\hline $\mathrm{A}_{2}$ & $1,17 \times 10^{4}$ & $8,30 \times 10^{-2}$ & 0,97 \\
\hline $\mathrm{A}_{3}$ & $1,19 \times 10^{4}$ & $2,90 \times 10^{-1}$ & 3,45 \\
\hline $\mathrm{A}_{4}$ & $1,18 \times 10^{4}$ & $4,06 \times 10^{-1}$ & 4,79 \\
\hline $\mathrm{A}_{5}$ & $1,19 \times 10^{4}$ & $4,92 \times 10^{-1}$ & 5,85 \\
\hline
\end{tabular}

norma ASTM D 1652-97 ${ }^{[19]}$. O agente de cura usado como co-monômero foi a poliamina alifática líquida trietilenotetramina (TETA), produto DEH 24 da Dow Química do Brasil com $30 \mathrm{~g} \mathrm{eq}^{-1} \mathrm{o}$ que foi determinado por três métodos analíticos que foram recentemente divulgados ${ }^{[20]}$.

Oito copolímeros aleatórios a base de acrilato de 2-etilhexila (AEH) e metacrilato de metila (MMA) contendo $80 \%$ (molar) de MMA foram sintetizados a $70{ }^{\circ} \mathrm{C}$ por técnica de polimerização em solução via radical livre usando tetrahidrofurano como solvente. Três desses copolímeros foram sintetizados usando ácido mercaptoacético como agente de transferência de cadeia o que permitiu sintetizar copolímeros com diferente massa molecular $\left(\mathrm{M}_{1}-\mathrm{M}_{3}\right.$ materiais listados na Tabela 1). O procedimento utilizado na síntese foi divulgado em trabalho anterior ${ }^{[11]}$. Os outros cinco copolímeros foram sintetizados por um procedimento semelhante sem adição do agente de transferência de cadeia usando 2,2'-azo-bis-isobutironitrila (AIBN) como iniciador e adicionando diferentes quantidades de um terceiro monômero que contem grupos carboxílicos (ácido acrílico) o que permitiu obter copolímeros com diferente concentração de grupos carboxílicos sem mudanças significativas na massa molecular $\left(\mathrm{A}_{1}-\mathrm{A}_{5}\right.$ materiais listados na Tabela 1).

\section{Preparação das misturas}

A modificação do monômero epoxídico foi realizada pela pré-reação da resina com os copolímeros $\mathrm{M}_{1}, \mathrm{M}_{2}$ e $\mathrm{M}_{3}$ como sistemas independentes usando sempre uma concentração de $10 \mathrm{phr}$ (phr: partes de copolímero por cada 100 partes de resina). O procedimento utilizado para a modificação do monômero epoxídico foi divulgado em trabalho anterior ${ }^{[11]}$. Para os copolímeros acrílicos $\mathrm{A}_{1}, \mathrm{~A}_{2}, \mathrm{~A}_{3}, \mathrm{~A}_{4}$ e $\mathrm{A}_{5}$ o procedimento de modificação foi diferente ao anterior. No entanto, foi utilizada a mesma concentração de copolímero (10 phr). Neste caso, o procedimento de modificação consistiu em dissolver a resina e o copolímero em diclorometano à temperatura ambiente. Após a solubilização dos componentes, o diclorometano foi removido cuidadosamente sob vácuo em duas etapas; primeiro à temperatura ambiente, e depois a $120^{\circ} \mathrm{C}$. Todos os adesivos modificados e o adesivo puro foram preparados à temperatura ambiente pela adição do endurecedor tanto à resina contendo o copolímero, como à resina pura, respec- 
tivamente. Foram usadas proporções estequiométricas entre os grupos funcionais e o programa de cura usado foi de duas etapas. A primeira etapa, 24 horas à temperatura ambiente, e a segunda, em condições isotérmicas que permitem alcançar a conversão máxima ${ }^{[21,22]}$.

\section{Análises calorimétricas}

Os sistemas modificados e o sistema puro foram estudados por experiências isotérmicas a $50{ }^{\circ} \mathrm{C}$ utilizando o analisador Perkin-Elmer DSC-7, sob atmosfera de nitrogênio e quantidades de amostra na faixa de 15 a $20 \mathrm{mg}$.

\section{Análises reológicas}

As análises reológicas dos adesivos líquidos foram realizadas num reômetro (Anton Paar-Physica MCR 301; Alemanha) de pratos paralelos de $25 \mathrm{~mm}$ de diâmetro e $0,5 \mathrm{~mm}$ de espessura. Os ensaios foram realizados em condições isotérmicas a $40{ }^{\circ} \mathrm{C}$ usando frequiência oscilatória constante de $1 \mathrm{~Hz}$. As propriedades viscoelásticas dos adesivos durante a cura foram monitoradas, por medidas da viscosidade dinâmica complexa $\left(\eta^{*}\right)$, do módulo de armazenamento (G'), e do módulo de perda (G”) como uma função do tempo.

\section{Resistência mecânica}

As propriedades de aderência ao cisalhamento foram avaliadas em juntas de aço-aço (liga API X60) através de ensaios de resistência mecânica usando juntas coladas de cisalhamento simples segundo a norma ASTM D 1002. Antes da aplicação do adesivo, a superfície do substrato foi desengordurada e tratada mecanicamente. $\mathrm{O}$ tratamento mecânico consistiu no jateamento abrasivo seco utilizando granalha de perfil angular comercial de aço GH 40B e uma pressão no jato de 7,0 $\mathrm{kgf} \mathrm{cm}^{-2}$. Depois do tratamento as peças de aço $(102 \times 25 \times 1,6 \mathrm{~mm})$ foram montadas como juntas de cisalhamento simples usando uma extensão de colagem de $312,5 \mathrm{~mm}^{2}$. Os ensaios foram conduzidos numa máquina universal de ensaios (Instron model 5569) com célula de carga de $10 \mathrm{kN}$ usando uma velocidade do travessão de $0,5 \mathrm{~mm} \mathrm{~min}^{-1}$. A resistência mecânica foi expressa em $\mathrm{MPa}$, que fora calculado pela divisão do valor da carga na ruptura pela área de colagem. Os ensaios foram conduzidos a $22{ }^{\circ} \mathrm{C}$ e os valores foram a media de 10 determinações.

\section{Micrografias eletrônicas de varredura (SEM)}

Os adesivos a base de resina modificada foram vazados em moldes de silicone e submetidos ao mesmo programa de cura em duas etapas mencionado anteriormente. Isto permitiu obter corpos de prova com dimensões de $63,0 \times 6,0 \times 2,0 \mathrm{~mm}^{3}$, os quais foram fraturados por ensaios de impacto tipo Izod à temperatura ambiente. As superfícies de fratura resultantes desses ensaios foram cobertas de uma fina camada de ouro e observadas num microscópio eletrônico Jeol-JMS-5300 operando entre 5 e $15 \mathrm{kV}$. Três micrografias de cada formulação foram analisadas utilizando o programa de imagens Global Lab o que permitiu determinar os diâmetros das par- tículas e construir as curvas de distribuição de tamanhos. O diâmetro médio das partículas foi determinado utilizando a Equação 1.

$$
\text { diâmetro médio }=\frac{\sum n_{\mathrm{i}} \mathrm{di}_{\mathrm{i}}}{\sum \mathrm{n}_{\mathrm{i}}}
$$

onde $\mathrm{n}_{\mathrm{i}}$ : representa o número de partículas i; e $\mathrm{d}_{\mathrm{i}}$ : representa o diâmetro da partícula i.

\section{Resultados e Discussão}

\section{Influência dos copolímeros na velocidade da reação de polimerização}

Para uma correta compreensão dos comportamentos morfológicos dos adesivos estudados neste trabalho, é importante dizer que a trietilenotetramina (TETA) é um agente de cura popularmente utilizado em formulações adesivas do tipo bicomponente. Este agente de cura é do tipo poliamina alifática onde na sua estrutura química existem átomos de nitrogênios do tipo amina primária e secundária. Particularmente nas formulações contendo a resina do tipo éter diglicidílico do Bisfenol-A (DGEBA) com trietilenotetramina, utiliza-se proporção não estequiométrica do agente de cura (70\% da quantidade estequiometrica $)^{[23]}$. Entretanto, para obter as melhores propriedades se utiliza proporção estequiométrica, devido a que nesta condição, o sistema consegue a estrutura de rede mais completa, já que alcança a conversão máxima.

Segundo a literatura o modelo de reação mais popular para esta formulação e o proposto por Horie ${ }^{[24]}$ onde se assume que a reação ocorre por dois mecanismos diferentes; um deles o mecanismo não-catalítico, e o outro o mecanismo auto-catalítico segundo é ilustrado na Figura 1. Ambos os mecanismos estão baseados em sucessivas reações de adição dos grupos epoxídicos com os grupos amina primária e
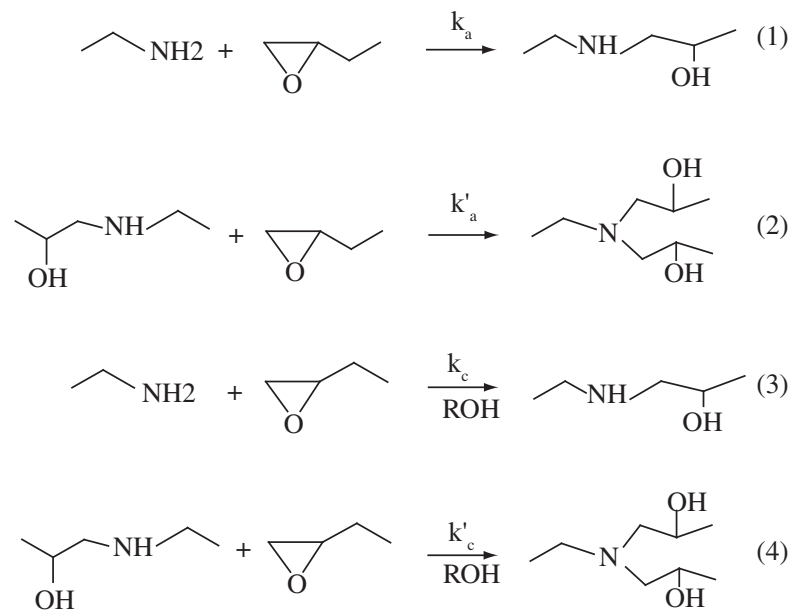

Figura 1. Mecanismos da reação do sistema de resina epoxídica do tipo éter diglicidílico do Bisfenol-A (DGEBA) com trietilenotetramina (TETA). $\mathrm{k}_{\mathrm{a}}$, $\mathrm{k}^{\prime}$ ' $\mathrm{k}, \mathrm{k}$ ' representam as constantes de velocidades relativas das reações de adição não-catalíticas e catalíticas que originam grupamentos amina do tipo secundário e terciário, respectivamente. 
secundária, via mecanismo não catalisado, e via mecanismo catalisado pelos grupos hidroxílicos, os quais estão presentes no início da reação, ou são formados nas reações de adição. Nestas reações de adição como representado na Figura 1 originam-se estruturas contendo grupamentos do tipo amina secundária (Figura 1, Equações 1 e 3) e amina terciária (Figura 1, Equações 2 e 4), e um grupo hidroxílico em cada reação de adição. Na Figura 1 as Equações 1 e 2 correspondem ao mecanismo não catalítico e as Equações 3 e 4 correspondem ao mecanismo catalítico.

Sabe-se que estes dois mecanismos são competitivos, que se diferenciam nos valores de energias de ativação $\left(E_{\mathrm{a}}\right)$. Para o catalítico $\mathrm{E}_{\mathrm{a}}=58,6 \mathrm{~kJ} \mathrm{~mol}^{-1}$, e para o não-catalítico $\mathrm{E}_{\mathrm{a}}=102 \mathrm{~kJ} \mathrm{~mol}^{-1}$. Que devido à diferença entre estes valores de energia de ativação, o primeiro predomina a temperaturas baixas, e o segundo predomina a temperaturas altas. Entretanto, o primeiro predomina em baixas conversões mesmo a temperaturas altas. Estes dois mecanismos de reação são típicos de sistemas epoxídicos que utilizam agentes de cura do tipo aminas alifáticas, onde geralmente são utilizadas proporções estequiométricas ou quase estequiométricas entre os grupos funcionais, o que garante que nesta condição não ocorre a reação de homopolimerização dos grupos epoxídicos, como acontece no caso de agentes de cura do tipo amina aromática. Sabe-se também que para o sistema que utiliza aminas primarias como agente de cura, a cinética de reação é de ordem 2, e que a velocidade da reação não fica comprometida durante a gelificação como ocorre em toda polimerização clássica via mecanismo por etapas. Entretanto, a velocidade da reação diminui quando a vitrificação é alcançada, tempo onde a reação pára, de maneira temporal, se a temperatura de cura utilizada for inferior à máxima temperatura onde o sistema vitrifica $\left(\operatorname{Tg}_{\infty}\right)$. Sabe-se ainda que a expressão cinética que descreve a reação de resina epoxídica com aminas alifáticas em condições estequiométricas assumindo o mecanismo proposto por Horie é dada pela Equação $2^{[25]}$.

$$
\left(\frac{\mathrm{dX} / \mathrm{dt}}{1-\mathrm{X}^{2}}\right)=\left[\mathrm{k}_{\mathrm{a}}+\mathrm{k}_{\mathrm{c}}\left([\mathrm{OH}]_{\mathrm{o}} /[\mathrm{e}]_{\mathrm{o}}+\mathrm{X}\right)\right]
$$

onde $\mathrm{X}$ : conversão da reação química; ka: constante de velocidade relativa do mecanismo não-catalítico; kc: constante de velocidade relativa do mecanismo catalítico; $[\mathrm{OH}]_{0}$ : concentração inicial de grupos hidroxílicos; e $[\mathrm{e}]_{0}$ : concentração inicial de grupos epoxídicos

A Figura 2 mostra os tempos de gelificação dos adesivos determinados a partir das análises reológicas em condições isotérmicas a $40{ }^{\circ} \mathrm{C}$, considerando como tempo de gelificação, o ponto de interseção entre as curvas do módulo de armazenamento (G') e do módulo de perda (G') colocadas como uma função do tempo de reação ${ }^{[26]}$. Observa-se que os tempos de gelificação não mudaram de modo significativo com o aumento da massa molecular ou com o aumento da concentração de grupos carboxílicos nos copolímeros. Uma explicação para este comportamento consiste em considerar que os copolímeros não participaram da reação de cura, o que significa que os grupos carboxílicos presentes nos co-

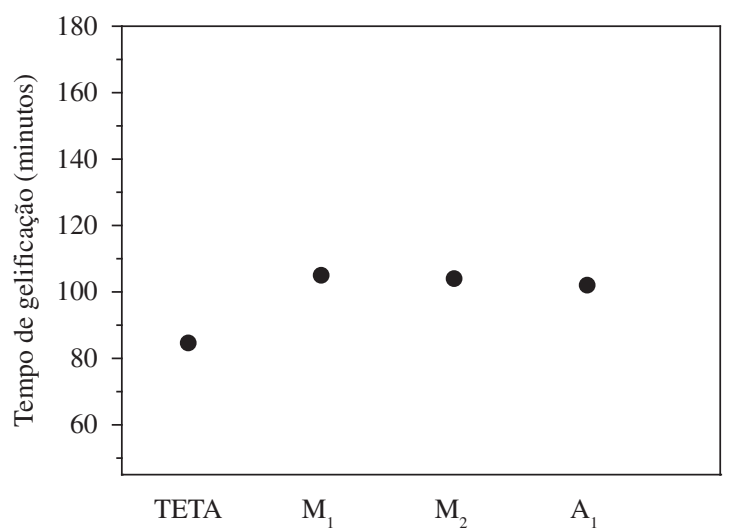

(a)

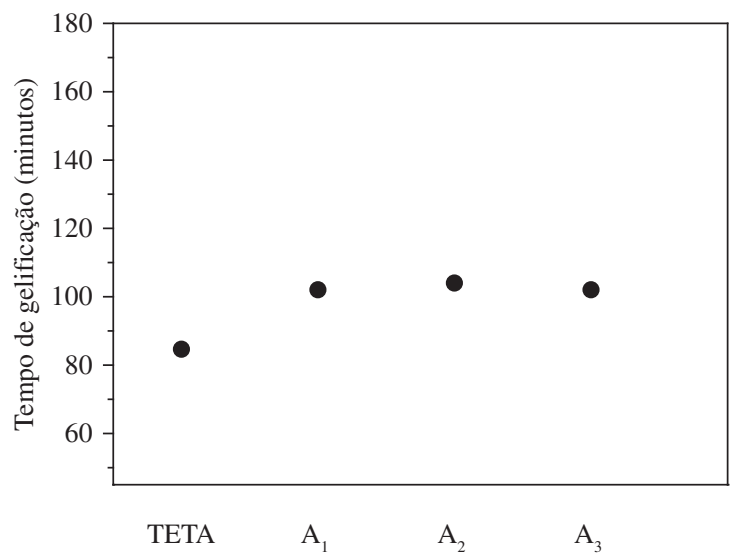

(b)

Figura 2. Tempos de gelificação determinados a $40{ }^{\circ} \mathrm{C}$, para o adesivo puro (TETA), e para os adesivos com 10 phr de copolímero acrílico; a) com diferente massa molecular; $\mathrm{M}_{1}\left(\overline{\mathrm{Mn}}=8,74 \times 10^{3}\right), \mathrm{M}_{2}\left(\overline{\mathrm{Mn}}=3,40 \times 10^{3}\right)$ e $\mathrm{A}_{1}$ $\left(\overline{\mathrm{Mn}}=1,19 \times 10^{4}\right)$; b) com diferente concentração de grupos carboxílicos; $\mathrm{A}_{1}$ $\left(0,00 \mathrm{mmol} \mathrm{g}^{-1}\right), \mathrm{A}_{2}\left(8,30 \times 10^{-2} \mathrm{mmol} \mathrm{g}^{-1}\right)$ e $\mathrm{A}_{3}\left(2,90 \times 10^{-1} \mathrm{mmol} \mathrm{g}^{-1}\right)$.

polímeros reagiram com os grupos epoxídicos na etapa de pré-reação do monômero epoxídico, e como conseqüência disto, os copolímeros parecem não afetar a velocidade da reação. Este comportamento está de acordo com o divulgado na literatura ${ }^{[1]}$, onde assegura-se que os grupos carboxílicos do modificador reagem com o monômero epoxídico.

De maneira comparativa as medidas do tempo de gelificação entre os adesivos modificados revelaram que a presença do copolímero provocou um pequeno efeito de retardação quando comparado ao adesivo puro. Isto foi atribuído, a que antes da separação de fases, ou seja, antes da precipitação do modificador ocorreu uma diminuição relativa na concentração dos grupos funcionais, o que é denominado na literatura como efeito de diluição ${ }^{[6,27]}$. Este comportamento confirmou que a massa molecular (Figura 2a) e a concentração de grupos carboxílicos (Figura 2b) não afetaram a velocidade da polimerização, considerando a existência de erros experimentais durante a determinação dos tempos de gelificação.

Uma informação adicional da influência dos copolímeros na velocidade da reação foi obtida utilizando a calorimetria diferencial de varredura (DSC). A Figura 3 compara as curvas de conversão versus tempo, relativas aos adesivos modifica- 


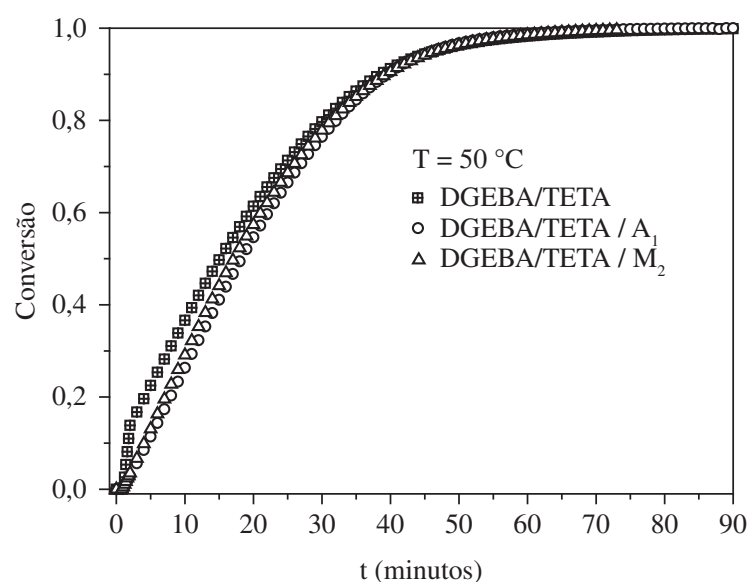

(a)

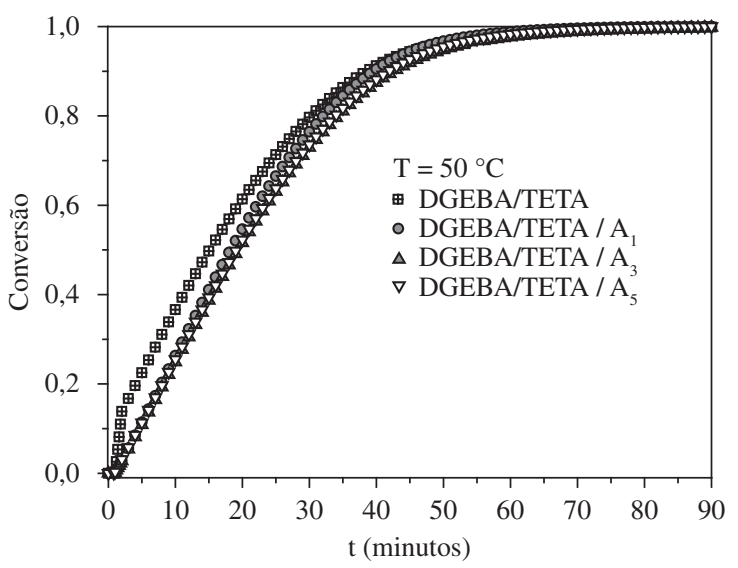

(b)

Figura 3. Curvas de conversão vs. tempo de reação obtidas por calorimetria diferencial de varredura (DSC) a partir de experiências isotérmicas a $50{ }^{\circ} \mathrm{C}$ para o adesivo puro, e para os adesivos com $10 \mathrm{phr}$ de copolímeros acrílicos. a) com diferente massa molecular; $\mathrm{M}_{2}\left(\overline{\mathrm{Mn}}=3,40 \times 10^{3}\right)$ e $\mathrm{A}_{1}$ $\left(\overline{\mathrm{Mn}}=1,19 \times 10^{4}\right) ; \mathrm{e}$ b) com diferente concentração de grupos carboxílicos; $\mathrm{A}_{1}\left(0,00 \mathrm{mmol} \mathrm{g}^{-1}\right), \mathrm{A}_{3}\left(2,90 \times 10^{-1} \mathrm{mmol} \mathrm{g}^{-1}\right)$ e $\mathrm{A}_{5}\left(4,92 \times 10^{-1} \mathrm{mmol} \mathrm{g}^{-1}\right)$.

dos, com aquela correspondente ao adesivo puro, obtidas por experiências de calorimetria isotérmica a $50{ }^{\circ} \mathrm{C}$. A conversão foi obtida pela Equação 3.

$$
\mathrm{X}=\frac{\left(\Delta H_{\mathrm{t}}\right)}{\Delta H_{\infty}}
$$

onde $\mathrm{X}$ representa a conversão no tempo $\mathrm{t} ; \Delta \mathrm{H}_{\mathrm{t}}$ : representa a entalpia parcial no tempo t; e $\Delta \mathrm{H}_{\infty}$ : representa a entalpia total da reação. Valores obtidos da área sob a curva calorimétrica.

Como observado na Figura 3, a incorporação dos copolímeros no adesivo epoxídico provocaram um ligeiro efeito de retardação cinética, e não ocorreram diferenças entre as velocidades de reação entre os adesivos modificados. Isto confirmou que a massa molecular do modificador (Figura 3a) e a concentração de grupos carboxílicos nos copolímeros (Figura 3b) não afetaram a velocidade da reação.

\section{Separação de fases e morfologia gerada}

Os adesivos modificados com os copolímeros acrílicos preparados à temperatura ambiente ficaram transparentes. Isto foi indicativo de que os copolímeros são solúveis no adesivo epoxídico. No entanto, depois de um tempo tornaram-se opacos, revelando-se o fenômeno de separação de fases. A Figura 4 mostra de modo comparativo, os tempos de separação de fases, determinados a partir das análises reológicas a $40{ }^{\circ} \mathrm{C}$ para os adesivos com os copolímeros de diferentes massas moleculares, e diferentes concentrações de grupos carboxílicos, onde foi incluído o tempo de gelificação do adesivo puro para fins comparativos.

O procedimento utilizado para a determinação do tempo de separação de fases, a partir das análises reológicas, foi baseado num procedimento divulgado na literatura ${ }^{[28,29]}$. O procedimento consiste em considerar que a separação de fases ocorre no momento onde, na curva viscosimétrica construída pela razão do valor de viscosidade dinâmica complexa, $(\eta *)$ e um valor arbitrário de viscosidade $\left(\eta_{0}\right.$, viscosidade de referencia) colocadas em função do tempo de reação, é observado um ligeiro aumento na viscosidade ("on set"). Depois deste ligeiro aumento da viscosidade ("on set") na curva

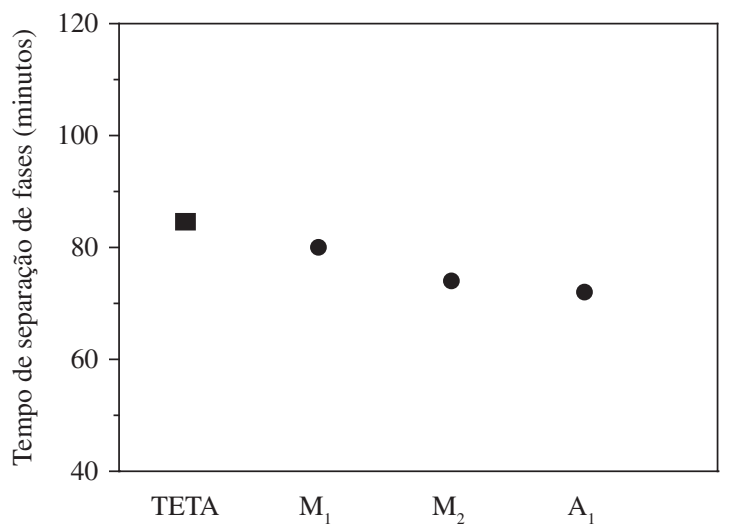

(a)

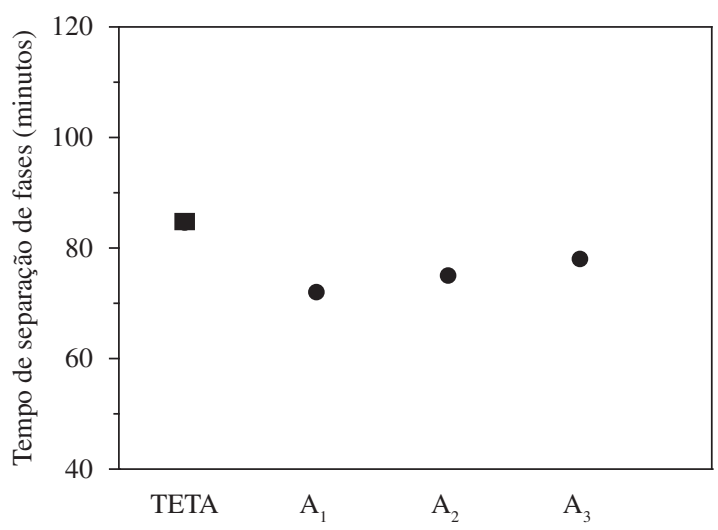

(b)

Figura 4. Tempo de gelificação para o adesivo puro (TETA) e tempo de separação de fases dos adesivos com 10 phr de copolímero acrílico. a) com diferente massa molecular; $\mathrm{M}_{1}(\overline{\mathrm{Mn}}=8,74 \times 103) ; \mathrm{M}_{2}(\overline{\mathrm{Mn}}=3,40 \times 103) ; \mathrm{A}_{1}$ $(\overline{\mathrm{Mn}}=1,19 \times 104)$; e b) com diferente concentração de grupos carboxílicos; $\mathrm{A}_{1}\left(0,00 \mathrm{mmol} \mathrm{g}^{-1}\right) ; \mathrm{A}_{2}\left(8,30 \times 10^{-2} \mathrm{mmol} \mathrm{g}^{-1}\right) ; \mathrm{e} \mathrm{A}_{3}\left(2,90 \times 10^{-1} \mathrm{mmol} \mathrm{g}^{-1}\right)$. 
viscosimétrica, a viscosidade diminui, devido à precipitação do modificador. Posteriormente a viscosidade se incrementa gradualmente, à medida que a reação ocorre, até alcançar a gelificação.

A separação de fases determinada experimentalmente ocorreu em tempos próximos quando os adesivos modificados são comparados, mas sempre este fenômeno ocorreu antes da gelificação do adesivo puro. Este comportamento coincide com aquele relatado por outros pesquisadores ${ }^{[2,4-6]}$, no estudo de sistemas epoxídicos que utilizam como agentes de cura aminas primárias, e anidrido de ácido, com poli(metacrilato de metila) como modificador.

O tempo de separação de fases apresentou uma ligeira e sistemática diminuição com o aumento da massa molecular do modificador (Figura 4a). Para o adesivo contendo o copolímero $\mathrm{A}_{1}$, que corresponde ao modificador de maior massa molecular, a separação de fases foi alcançada em menor tempo. Este comportamento indicou que o aumento da massa molecular induz para que a separação de fases ocorra numa conversão mais baixa. Esta afirmação está fundamentada nos dados obtido neste trabalho e em um trabalho anterior ${ }^{[9]}$, onde a massa molecular do modificador não apresentou mudança na velocidade da reação. Por outro lado, a separação de fases apresentou uma ligeira e sistemática retardação com o aumento da concentração de grupos carboxílicos (Figura 4b). Para o adesivo contendo o copolímero $\mathrm{A}_{1}$, que corresponde ao modificador que não possui grupos carboxílicos, a separação de fases foi alcançada em menor tempo. Este comportamento indicou que o aumento da concentração de grupos carboxílicos no copolímero induz para que a separação de fases ocorra numa conversão mais alta. Estas afirmações estão fundamentadas nos dados obtidos neste trabalho, onde as concentrações de grupos carboxílicos dos modificadores não apresentaram mudanças na velocidade da reação. Estes resultados coincidem com o relatado na literatura ${ }^{[1,30,31]}$ no modelo que utiliza a equação de Flory-Huggins para descrever o fenômeno de separação de fases induzido por reação química.

A Figura 5 mostra as micrografias eletrônicas de varredura (SEM) correspondentes à morfologia gerada após o programa de cura, em duas etapas, para os adesivos com os copolímeros acrílicos de diferentes massas moleculares. A morfologia gerada é bifásica do tipo fase dispersa/matriz, onde aparece uma fase contínua com regiões estriadas, que inclui também regiões lisas, e outra fase dispersa na forma de partículas esféricas de cor branca ou preta. As partículas de cor branca foram atribuídas à fase do copolímero acrílico, e as de cor preta foram atribuídas a buracos que ocorrem como conseqüência do desprendimento das partículas do copolímero na matriz durante a fratura.

Nas micrografias da Figura 5 se observou que os tamanhos das partículas da fase dispersa aumentaram com o aumento da massa molecular do modificador. Por exemplo, quando a massa molecular aumentou de $3,40 \times 10^{3}\left(\mathrm{M}_{2}\right)$ para $1,19 \times 10^{4}$ $\left(\mathrm{A}_{1}\right)$, o diâmetro médio das partículas também aumentou de um valor inferior a 0,10 para $0,32 \mu \mathrm{m}$. Este comportamento

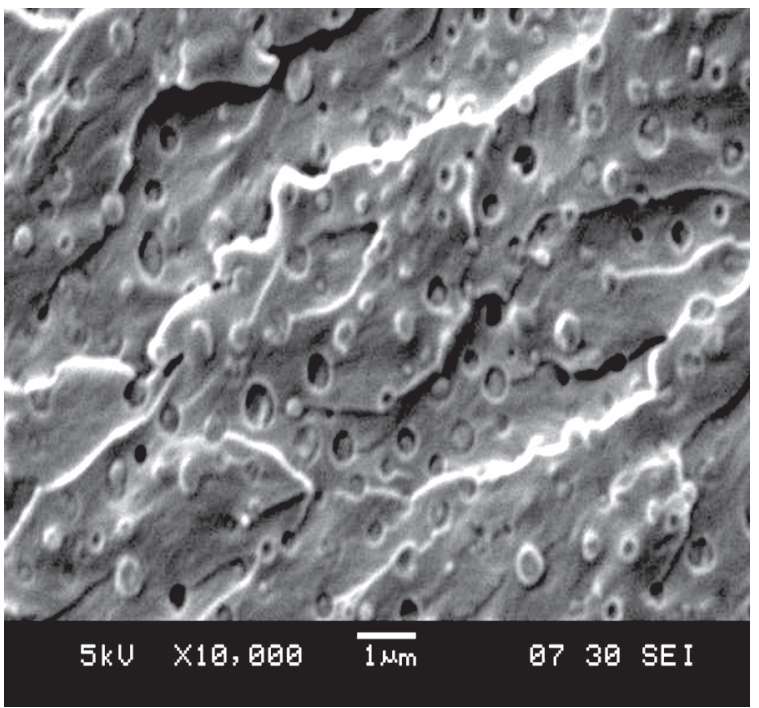

$\left(\mathrm{A}_{1}\right)$

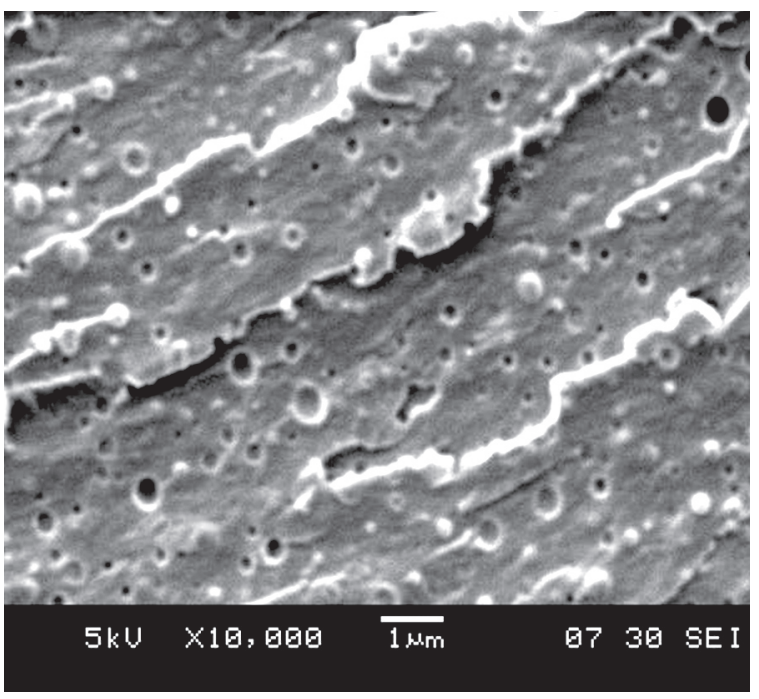

$\left(\mathrm{M}_{1}\right)$



$\left(\mathrm{M}_{2}\right)$

Figura 5. Micrografias eletrônicas (SEM) da superfície de fratura dos adesivos com diferente massa molecular; $\mathrm{A}_{1}\left(\overline{\mathrm{Mn}}=1,19 \times 10^{4}\right), \mathrm{M}_{1}\left(\overline{\mathrm{Mn}}=8,74 \times 10^{3}\right)$ e $\mathrm{M}_{2}\left(\overline{\mathrm{Mn}}=3,40 \times 10^{3}\right)$ após o programa de cura de duas etapas. 


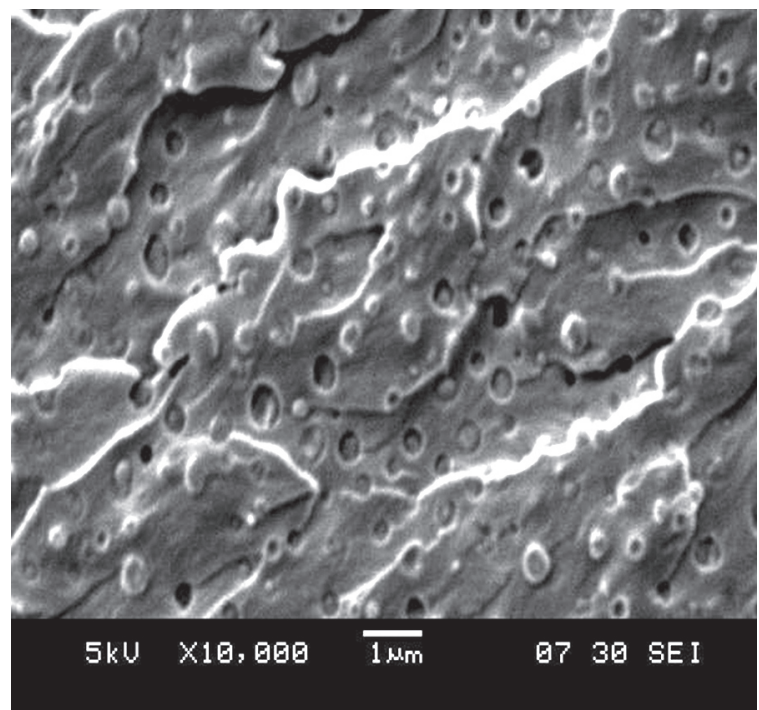

$\left(\mathrm{A}_{1}\right)$

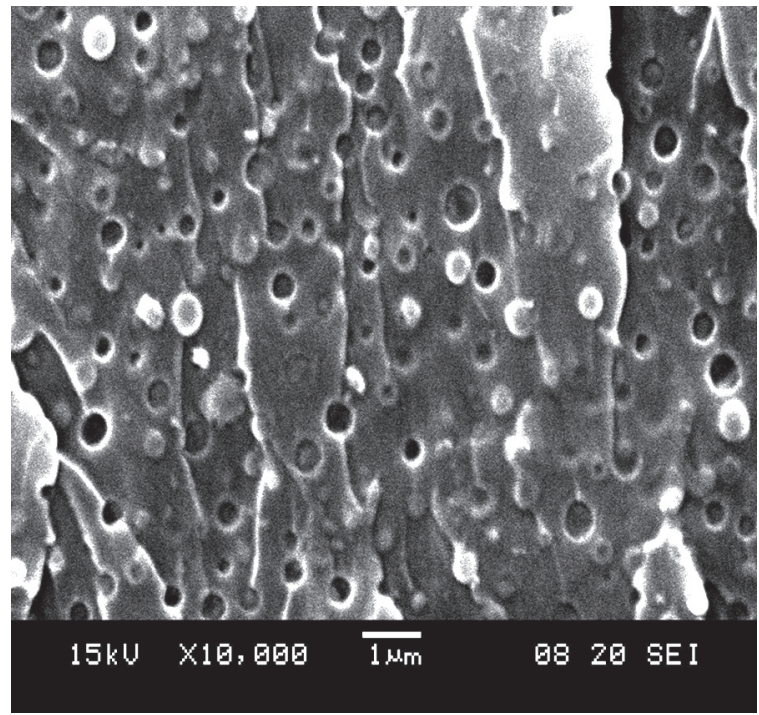

$\left(\mathrm{A}_{2}\right)$

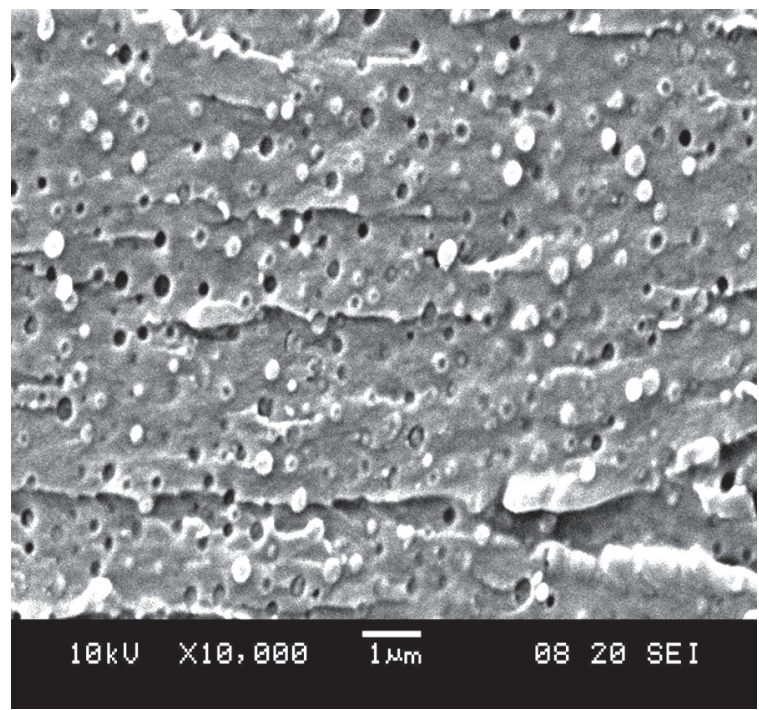

$\left(\mathrm{A}_{3}\right)$

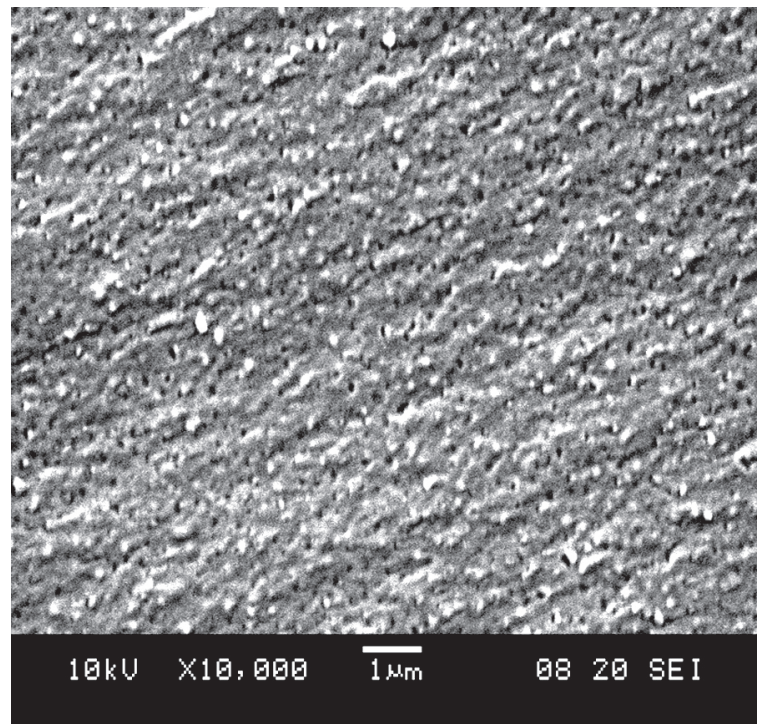

$\left(\mathrm{A}_{4}\right)$

Figura 6. Micrografias eletrônicas (SEM) da superfície de fratura dos adesivos com diferente concentração de grupos carboxílicos; $\mathrm{A}_{1}\left(0,00 \mathrm{mmol} \mathrm{g}^{-1}\right)$; $\mathrm{A}_{2}\left(8,30 \times 10^{-2} \mathrm{mmol} \mathrm{g}^{-1}\right) ; \mathrm{A}_{3}\left(2,90 \times \mathrm{1}^{-1} \mathrm{mmol} \mathrm{g}^{-1}\right) ; \mathrm{e}_{4}\left(4,06 \times 10^{-1} \mathrm{mmol} \mathrm{g}^{-1}\right)$ após o programa de cura de duas etapas.

morfológico indicou que o aumento da massa molecular do copolímero induz para que a separação de fases ocorra a uma conversão mais baixa, o que significa que a separação de fases aconteceu a uma viscosidade mais baixa, o que favoreceu o crescimento das partículas. Este comportamento corroborou que a massa molecular do copolímero não afetou a velocidade da reação. Estas considerações, e o comportamento experimental observado para os domínios dos copolímeros com diferentes massas moleculares, coincide com o relatado para outros sistemas epoxídicos modificados, tanto com elastômeros, como com termoplásticos ${ }^{[1]}$.

A influência da concentração dos grupos carboxílicos na morfologia gerada é apresentada na Figura 6, onde são mostradas as micrografias eletrônicas (SEM) correspondentes a cada adesivo, depois do programa de cura em duas etapas. Neste caso, para uma melhor observação do comportamento



Figura 7. Influência da concentração de grupos carboxílicos na curva de distribuição de tamanhos para os adesivos com $10 \mathrm{phr}$ de copolímero acrílico; $\mathrm{A}_{2}\left(8,30 \times 10^{-2} \mathrm{mmol} \mathrm{g}^{-1}\right) ; \mathrm{A}_{3}\left(2,90 \times 10^{-1} \mathrm{mmol} \mathrm{g}^{-1}\right) ; \mathrm{e}_{4}\left(4,06 \times 10^{-1} \mathrm{mmol} \mathrm{g}^{-1}\right)$ após o programa de cura de duas etapas. 
morfológico foram construídas as curvas de distribuição de tamanhos, o que é ilustrado na Figura 7.

Nas Figuras 6 e 7 observou-se que o aumento da concentração de grupos carboxílicos no copolímero acrílico conduz à formação de uma fase dispersa de menor tamanho. Isto ficou mais claro na Figura 7, onde para altas concentrações de grupos carboxílicos no copolímero ocorreu o deslocamento significativo da curva de distribuição para menores tamanhos, e a distribuição tendeu a ser mais estreita. Este comportamento morfológico, onde o aumento da concentração de grupos funcionais acarreta uma diminuição do tamanho dos domínios, é resultado de um aumento de solubilidade do modificador no sistema epoxídico, o que provoca que a separação de fases ocorra em tempos mais longos, onde a viscosidade é mais alta, e como conseqüência disto, o crescimento dos domínios fica limitado. Isto assegura que seja originada uma fase dispersa com tamanhos de partículas mais uniformes e de menor tamanho.

\section{Influência da massa molecular do modificador na aderência ao cisalhamento}

A resistência mecânica das juntas coladas produzidas com os adesivos modificados, contendo os copolímeros de diferente massa molecular é mostrada na Figura 8. A resistência mecânica mudou de maneira significativa, ainda para valores de massas moleculares da mesma ordem de grandeza. Isto foi indicativo de que a resistência à ruptura ao cisalhamento é bem sensível a pequenas mudanças de massa molecular no copolímero.

Como observado na Figura 8, a aderência ao cisalhamento inicialmente aumentou até alcançar um valor máximo para o adesivo com o copolímero $\mathrm{M}_{2}\left(\overline{\mathrm{Mn}}=3,40 \times 10^{3}\right)$ e depois diminui significativamente. $\mathrm{O}$ valor máximo de resistência à ruptura foi $13,7 \pm 0,3 \mathrm{MPa}$ para o adesivo com o copolímero $\mathrm{M}_{2}$ e o valor mínimo foi de $11,1 \pm 0,5 \mathrm{MPa}$ para o adesivo com o copolímero $\mathrm{M}_{3}\left(\overline{\mathrm{Mn}}=2,10 \times 10^{3}\right)$. Para relacionar as propriedades adesivas à morfologia gerada foram produzidos corpos de prova, os quais foram fraturados por ensaios de impacto tipo Izod e as superfícies de fratura

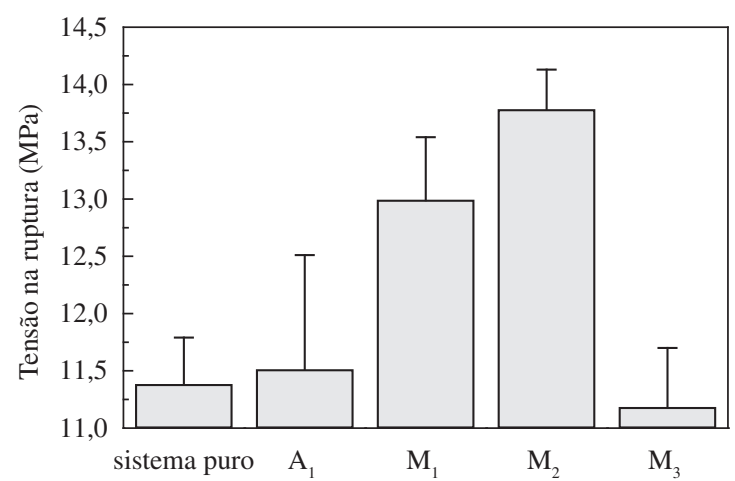

Figura 8. Influência da massa molecular dos copolímeros na tensão de ruptura ao cisalhamento em juntas de aço-aço; $\mathrm{A}_{1}\left(\overline{\mathrm{Mn}}=1,19 \times 10^{4}\right), \mathrm{M}_{1}$ $\left(\overline{\mathrm{Mn}}=8,74 \times 10^{3}\right) \mathrm{M}_{2}\left(\overline{\mathrm{Mn}}=3,40 \times 10^{3}\right) \mathrm{e}_{3}\left(2,10 \times 10^{3}\right)$ após o programa de cura de duas etapas. desses corpos foram analisadas usando microscopia eletrônica de varredura (SEM). As micrografias são mostradas na Figura 5.

$\mathrm{Na}$ Figura 5 observou-se que, quando a massa molecular do modificador diminuiu, o diâmetro médio de partículas também diminuiu. Por exemplo, quando a massa molecular diminui de $1,19 \times 10^{4}\left(\mathrm{~A}_{1}\right)$ para $3,40 \times 10^{3}\left(\mathrm{M}_{2}\right)$, o diâmetro médio diminui de $0,32 \mu \mathrm{m}$ para um valor inferior a $0,10 \mu \mathrm{m}$. O melhor desempenho mecânico do adesivo com o copolímero $\mathrm{M}_{2}\left(\overline{\mathrm{Mn}}=3,40 \times 10^{3}\right)$ foi relacionado às características morfológicas baseadas em partículas com diâmetro médio menor a $0,10 \mu \mathrm{m}$, distribuídas de maneira homogênea na matriz termorrígida. Este comportamento está de acordo com o divulgado na literatura ${ }^{[1,32]}$, onde diz que para propósitos de tenacificação de polímeros termorrígidos resulta mais efetivo utilizar modificadores elastoméricos funcionalizados, de baixa massa molecular, os que originam uma fase dispersa baseada em partículas de pequeno tamanho. Estes sistemas estão caracterizados pela diminuição da $\mathrm{T} g$ do polímero termorrígido, onde o principal mecanismo de tenacificação envolve a cavitação interna das partículas, e a subseqüente formação de bandas de cisalhamento.

\section{Efeito da concentração de grupos carboxílicos na aderência ao cisalhamento}

A resistência mecânica das juntas coladas produzidas com os adesivos modificados usando os copolímeros com diferente concentração de grupos carboxílicos é mostrada na Figura 9. Inicialmente a resistência mecânica aumentou até alcançar um valor máximo quando a concentração de grupos carboxílicos foi de $8,3 \times 10^{-2} \mathrm{mmol} \mathrm{g}^{-1}$ (copolímero $\mathrm{A}_{2}$ ) e depois diminuiu. $\mathrm{O}$ máximo valor de resistência foi de $13,7 \pm 0,5 \mathrm{MPa}$ para o adesivo com o copolímero $\mathrm{A}_{2}$. Como ilustrado na Figura 9, quando a concentração de grupos carboxílicos aumentou, o diâmetro das partículas geradas diminuiu. Este comportamento morfológico era esperado, devido a que um aumento da concentração de grupos carboxílicos no copolímero, aumenta a solubilidade do copolímero no sistema epoxídico, e como conseqüência disto, a separação de

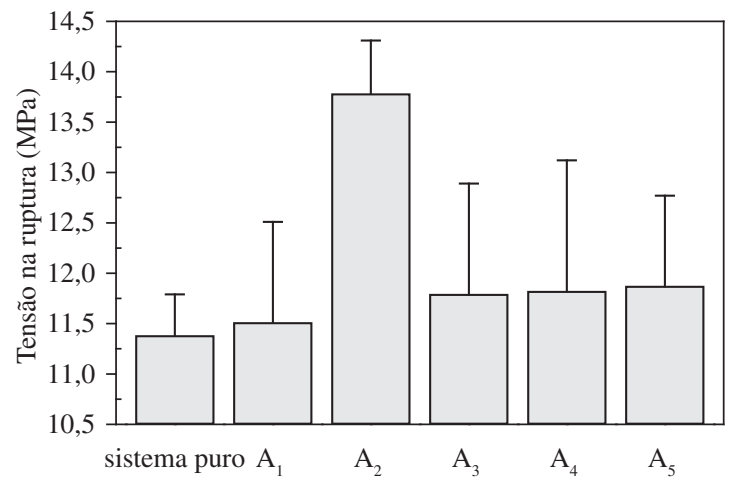

Figura 9. Influência da concentração de grupos carboxílicos nos copolímeros na tensão de ruptura ao cisalhamento em juntas de aço-aço; $\mathrm{A}_{1}\left(0,00 \mathrm{mmol} \mathrm{g}{ }^{-1}\right) ; \mathrm{A}_{2}\left(8,30 \times 10^{-2} \mathrm{mmol} \mathrm{g}^{-1}\right) ; \mathrm{A}_{3}\left(2,90 \times 10^{-1} \mathrm{mmol} \mathrm{g}^{-1}\right)$; $\mathrm{A}_{4}\left(4,06 \times 10^{-1} \mathrm{mmol} \mathrm{g}^{-1}\right)$; e $\mathrm{A}_{5}\left(4,92 \times 10^{-1} \mathrm{mmol} \mathrm{g}^{-1}\right)$ após o programa de cura de duas etapas. 
fases fica retardada e, portanto, os diâmetros das partículas geradas são menores. Isto foi discutido no item separação de fases e morfologia.

$\mathrm{O}$ melhor desempenho do adesivo com o copolímero $\mathrm{A}_{2}$ $\left(8,3 \times 10^{-2} \mathrm{mmol} \mathrm{g}^{-1}\right.$ de grupos carboxílicos) como modificador foi relacionado às características morfológicas baseadas numa distribuição de partículas com diâmetros na faixa de 0,20 a $0,52 \mu \mathrm{m}$, distribuídas de maneira homogênea na matriz. Neste caso, as partículas de menor tamanho não foram as que proporcionaram o melhor comportamento mecânico. Isto é conseqüência do tipo de partículas utilizadas, da forte adesão entre as partículas e a matriz, e do mecanismo de tenacificação envolvido. Neste caso, o melhor desempenho mecânico alcançado pela matriz termorrígida modificada foi usando partículas dispersas de um polímero termoplástico funcionalizado $\left(\overline{\mathrm{Mn}}=1,17 \times 10^{4}\right)$. Este tipo de modificadores não levam à diminuição da $\mathrm{T} g$ da matriz termorrígida, e o mecanismo de tenacificação mais importante é o chamado mecanismo de propagação de trincas (crack bridging mechanism $)^{[1,33]}$.

\section{Conclusões}

O sistema do éter diglicidílico do Bisfenol-A com trietilenotetramina modificado com diferentes copolímeros acrílicos foi estudado. Verificou-se que, os copolímeros acrílicos de diferentes massas moleculares e de diferentes concentrações de grupos carboxílicos mostraram mudanças significativas na morfologia gerada. As análises reológicas evidenciaram que os copolímeros acrílicos provocaram ligeiras mudanças para a observação da separação de fases, não mudaram de maneira significativa os tempos de gelificação e não foram observadas mudanças na velocidade de polimerização. $\mathrm{O}$ uso da calorimetria diferencial de varredura mostrou que os copolímeros como modificadores apresentaram o efeito de retardação cinética. Os resultados morfológicos e de aderência ao cisalhamento nas juntas coladas mostraram que, uma morfologia baseada em partículas com diâmetro médio menor a $0,10 \mu \mathrm{m}$, distribuídas de maneira homogênea na matriz termorrígida, apresentaram o melhor desempenho mecânico com o adesivo modificado com o oligômero de massa molecular $\overline{\mathrm{Mn}}=3,40 \times 10^{3}$. Entretanto, uma morfologia com uma distribuição de tamanhos de partículas na faixa de 0,20 a $0,52 \mu \mathrm{m}$, distribuídas de maneira homogênea na matriz mostraram o melhor desempenho na aderência ao cisalhamento com o adesivo contendo o copolímero acrílico com uma concentração de grupos carboxílicos de $8,3 \times 10^{-2} \mathrm{mmol} \mathrm{g}^{-1}$.

\section{Agradecimentos}

Os autores agradecem o apoio pelo Plano Nacional de Ciência e Tecnologia do Setor Petróleo e Gás Natural - CTPETRO, por meio do CNPq (CT-PETRO/CNPq) Proc. No. 500092/02-8.

\section{Referências Bibliográficas}

1. Williams, R. J. J.; Rozenberg, B. A. \& Pascault, J. P. Adv. Polym. Sci., 128, p.95 (1997).

2. Gómez, C. M. \& Bucknall, C. B. - Polymer, 34, p.2111 (1993).

3. Mondragon, I.; Remiro, P. M.; Martin, M. D.; Valea, A.; Franco, M. \& Bellenguer V. - Polym. Int., 47, p.152 (1998).

4. Galante, M. J.; Oyanguren, P. A.; Andromaque, K.; Frontini, P. M. \& Williams, R. J. J. - Polym. Int., 48, p.642 (1999).

5. Remiro, P. M.; Riccardi, C. C.; Corcuera, M. A. \& Mondragon, I. - J. Appl. Polym. Sci., 74, p.772 (1999).

6. Ritzenthaler, S.; Girard-Reydet, E. \& Pascault, J. P. Polymer, 41, p.6375 (2000).

7. Remiro, P. M.; Marieta, C.; Riccardi, C. C. \& Mondragon, I. - Polymer, 42, p.9909 (2001).

8. Stefani, P. M.; Riccardi, C. C.; Remiro, P. M.; \& Mondragon, I. - Polym. Ing. Sci. 41, p.2013 (2001).

9. González Garcia, F. \& Soares B. G. - Polímeros Ciência e Tecnologia, 13, p.235 (2003).

10. Cabanelas J. C.; Serrano, B. \& Baselga J. - Macromolecules, 38, p.961 (2005).

11. González Garcia, F. \& Soares, B. G. - "Toughened epoxy adhesives for the petroleum industry". in: Anais do X Colóquio Internacional de Macromoléculas, Gramado - RS, 10 -13 de abril de 2005.

12. Rezaifard, A. H.; Hodd, K. A.; Tod, D. A. \& Barton, J. M. - Int. J. Adhesion and Adhesives, 14, p.153 (1994).

13. Ochi, M. \& Shinzi, S. - Polymer, 40, p.1667 (1999).

14. Ritzenthaler, S.; Court, F.; David, L.; Girard-Reydet, E.; Leibler, L. \& Pascault, J. P. - Macromolecules, 35, p.3245 (2002).

15. Woo, E. M. \& Wu, M. N. - Polymer, 37, p.2485 (1996).

16. González Garcia, F.; Pires M. A. L.; Rodrigues Jr F. C. \& Sampaio E. M. - "Epoxy adhesive modified with 2-ethylhexyl acrylate-methyl methacrylate copolymer 3. Influence of epoxy hardener on adhesive properties". in: Anais do Congresso Mundial de Macromoléculas, Macro 2006, $41^{\circ}$ Simpósio Internacional de Macromoléculas, Rio de Janeiro - RJ, Julio de 2006.

17. Larrañaga, M.; Gabilongo, N.; Kortaberria, G.; Serrano, E., Remiro, P.; Riccardi, C. C. \& Mondragon, I. - Polymer 46, p.7082 (2005).

18. Fine T. \& Pascault J. P. - "Structured thermoplastic/thermoset blends using block copolymers". in: Anais do Congresso Mundial de Macromoléculas, Macro 2006, $41^{\circ}$ Simpósio Internacional de Macromoléculas, Rio de Janeiro - RJ, Julio de 2006. 
19. American Society for Testing and Materials. ASTM D 1652 - 97. "Standard test methods for epoxy content of epoxy resins" (1997).

20. González Garcia, F.; Silva, P. M.; Soares, B. G. \& Rieumont, J. - Polymer Testing, 26, p.95 (2007).

21. González Garcia, F.; Miguez, E. \& Soares, B. G. - Polímeros: Ciência e Tecnologia, 5, p.261 (2005).

22. González Garcia, F.; Soares, B. G.; Pita, V. J. R. R.; Sánchez, R. \& Rieumont, J. - Mechanical properties of epoxy networks based on DGEBA and aliphatic amines. Journal of Applied Polymer Science, aceito em 31 de março de 2006.

23. Lee, H. \& Neville, K. "Handbook of Epoxy Resins", McGraw-Hill, Inc. New York, (1967).

24. Horie, K.; Hiura, H.; Sawada, M.; Mita, I. \& Kambe, H. - J. Polym. Sci. (A-1) 8 p.1357 (1970).

25. Riccardi, C. C.; Adabbo, H. E. \& Williams R. J. J. - J. Appl. Polym. Sci. 29, p.2481 (1984).

26. Ampudia, J.; Larrauri, E.; Gil E. M.; Rodriguez, M. \& León, L. M. - J. Appl. Polym. Sci., 71, p.1239 (1999).
27. Martinez, I.; Martin, M. D.; Eceiza, A.; Oyanguren, P. \& Mondragon, I. - Polymer, 41, p.1027 (2000).

28. Choe, Y. \& Kim W. - Macromolecular Research, 10, p.259 (2002).

29. Choe, Y.; Kim, M. \& Kim W. - Macromolecular Research, 11, p.267 (2003).

30. Williams, R. J. J.; Borrajo, J.; Adabbo, H. E. \& Rojas, A. J. - "A Model for Phase Separation During a Thermoset Polymerization", in: Rubber-Modified Thermoset Resin, Adv. Chem. Ser. 208, (Ed. Riew K. and Gillham J.K.); American Chemical Society, Washington DC, p.195 (1984).

31. Vázquez, A.; Rojas, A. J.; Adabbo, H. E.; Borrajo, J. \& Williams, R. J. J. - Polymer, 28, p.1156 (1987).

32. Pearson, R. A. \& Yee, A. F. - Journal Materials Science 26, p.3828 (1991).

33. Cardwell, B. J. \& Yee, A. F. - Polym. Mat. Sci. Eng. (ACS), 70, p.254 (1994).

Enviado: $26 / 04 / 07$

Reenviado: 20/06/07

Aceito: $14 / 08 / 07$ 